\title{
Horizontal transfers of Mariner transposons between mammals and insects
}

\author{
Sarah G Oliveira ${ }^{1 *}$, Weidong Bao ${ }^{2}$, Cesar Martins ${ }^{1}$ and Jerzy Jurka ${ }^{2}$
}

\begin{abstract}
Background: Active transposable elements (TEs) can be passed between genomes of different species by horizontal transfer $(\mathrm{HT})$. This may help them to avoid vertical extinction due to elimination by natural selection or silencing. HT is relatively frequent within eukaryotic taxa, but rare between distant species.

Findings: Closely related Mariner-type DNA transposon families, collectively named as Mariner-1_Tbel families, are present in the genomes of two ants and two mammalian genomes. Consensus sequences of the four families show pairwise identities greater than 95\%. In addition, mammalian Mariner1_BT family shows a close evolutionary relationship with some insect Mariner families. Mammalian Mariner1_BT type sequences are present only in species from three groups including ruminants, tooth whales (Odontoceti), and New World leaf-nosed bats (Phyllostomidae).

Conclusions: Horizontal transfer accounts for the presence of Mariner_Tbel and Mariner1_BT families in mammals. Mariner_Tbel family was introduced into hedgehog and tree shrew genomes approximately 100 to 69 million years ago (MYA). Most likely, these TE families were transferred from insects to mammals, but details of the transfer remain unknown.
\end{abstract}

Keywords: DNA transposon, Genome evolution, Horizontal transfer, Mariner

\section{Findings}

In contrast to the vertical transmission of the genetic material from parents to offspring, the horizontal transfer $(\mathrm{HT})$ is a process in which new genetic information is transmitted between different, sometimes distant, species $[1,2]$. HT is likely to be one of the factors leading to the persistence of transposable elements (TEs) in eukaryotes [3-5], and complicating the evolutionary trees.

The detection of HT is mostly inferential, mainly based on the combination of two types of evidence: unusually high similarity between TE sequences from species that have long diverged from each other, and a limited distribution of one particular TE family within a group of species [6]. To date, numerous HTs have been detected in eukaryotes [6-10], but of particular interest are HTs across distant branches. A recent example of such a rare event is HT of hAT DNA transposon families between vertebrate and invertebrate species [11].

\footnotetext{
* Correspondence: sarahg.oliveira@gmail.com

${ }^{1}$ Morphology Department, Bioscience Institute, UNESP - Sao Paulo State University, Botucatu, Sao Paulo 18618-970, Brazil

Full list of author information is available at the end of the article
}

Here, we report two families of Mariner-type DNA transposons that have possibly undergone HT from insects to mammals. The first family, called Mariner_Tbel, was originally identified in the tree shrew (Tupaia belangeri), but families nearly identical to Mariner_Tbel were also found in the genome of another mammal, European hedgehog (Erinaceus europaeus), and in two ant species: red harvester ant (Pogonomyrmex barbatus) and Jerdon's jumping ant (Harpegnathos saltator) (Tables 1 and 2). Although the copy numbers and divergence vary between the families, the family consensus sequences reconstructed in each genome show a high level of identity to each other throughout the entire length (approximately $1.3 \mathrm{~kb}$ ) (Table 1). The lowest identity is found between the two families in E. europaeus and ant $P$. barbatus (95.84\%), and the highest identity is found between the two ant families (98.45\%). Therefore, unless otherwise stated, the four families in the genomes are referred collectively to as Mariner_Tbel families. Given the long divergence time between insects and mammals (approximately 1 billion years) [12-14], this high identity strongly indicates that HT took place during the evolutionary history of Mariner_Tbel families.

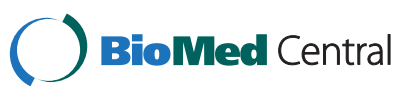


Table 1 Pairwise identities (\%) between the Mariner_Tbel consensus from mammals (Tupaia belangeri, Erinaceus europaeus) and insects (Pogonomyrmex barbatus, Harpegnathos saltator)

\begin{tabular}{llll}
\hline & T. belangeri & E. europaeus & P. barbatus \\
\hline E. europaeus & 96.55 & - & - \\
P. barbatus & 98.05 & 95.84 & - \\
H. saltator & 97.90 & 95.69 & 98.45 \\
\hline
\end{tabular}

This notion is consistent with the fact that mammal Mariner_Tbel sequences were found only in two distantly related mammalian species, even though over 30 mammalian genomes were sequenced to date.

We then estimated the approximate ages of the four Mariner_Tbel families in each genome. In mammals, we compared the sequence divergences of Mariner_Tbel to an older Mariner-type family (TIGGER1), relatively common in the mammalian genomes (Table 2). TIGGER1 elements are present in multiple copies in eutherian mammals, but only one or two degenerated copies were found in marsupial genomes, including Macropus eugenii, Monodelphis domestica, and Sarcophilus harrisii (Figure 1A). Therefore, mammalian TIGGER1 families likely expanded after the split of marsupials and placentals (190 million years ago (MYA)), but before the placental radiation (approximately $100 \mathrm{MYA}$ ) [15]. In the genome of the tree shrew and European hedgehog the divergence of the TIGGER1 family is $21.2 \pm 2 \%$ and $28.0 \pm 3 \%$, respectively (Table 2 ). Therefore, based on the divergence of Mariner_Tbel in the two mammal genomes $(15.0 \pm 2 \%$ and $19.4 \pm 3 \%$, respectively), the ages of Mariner_Tbel in the two mammals were calculated to be approximately 134 to 70 MYA and approximately 131 to 69 MYA, respectively. Because it is unlikely that the mammalian Mariner_Tbel expanded in the common ancestor of placental mammals before $100 \mathrm{MY}$, we adjusted the ages to be 100 to $70 \mathrm{MYA}$ and 100 to 69 MYA, respectively (see Figure 1A and [15]).

In the ant genomes, no Mariner family was yet identified as unambiguously present in the common ancestor of all ant species. Among potential candidates are the oldest known Mariner families present in some of the ant genomes (for example, Mariner-28_SIn or Mariner94_HSal; Figure 1). These small families may have expanded in the common ancestor of all ant species (140 MYA) [13], assuming that they were lost in some ant species. Alternatively, these old families might have expanded in some ant species after they split from their common ancestor. Under either scenario, the outermost ages when the two ant Mariner_Tbel families expanded could be still estimated by comparing their diversities with the diversity of Mariner-28_SIn (Table 2). Based on that, Mariner_Tbel family in the red harvester ant $(P$. barbatus) and Jerdon's jumping ant (H. saltator) expanded at most approximately 43 and approximately 50 million years ago, respectively.

The above age estimates suggest that the two ant Mariner_Tbel families are possibly younger than the mammalian Mariner_Tbel families. However, the history of Mariner_Tbel can be traced further back in ants and their insect relatives than in mammals. Individual Mariner_Tbel-like elements from distinct families, such as AEAQ01009575, AEAB01001421 and AFJA01006902 (Figure 1B), were also found in the genomes of two other ants (Solenopsis invicta and Camponotus florida$n u s$ ) as well as in the alfalfa leafcutting bee (Megachile rotundata). These Mariner_Tbel-like sequences and Mariner_Tbel sequences form a single lineage in the phylogenetic tree (Figure 1B), with the bee sequences in a more ancestral position (Figure 1B). The topology of this particular lineage mirrors the evolutionary history of

Table 2 Divergence of Mariner transposable element (TE) families in mammalian and insect genomes

\begin{tabular}{llll}
\hline Family & Length (bp) & Copy no. & Divergence (\%) $^{\mathbf{a}}$ \\
\hline Mariner-1_Tbel (TBel) & 1,279 & $>400$ & $15.0 \pm 2(183)$ \\
Mariner-1_Tbel (EEr) & 1,266 & $>70$ & $19.4 \pm 3(34)$ \\
Mariner-1_Tbel (PBa) & 1,285 & $>90$ & $6.3 \pm 1(53)$ \\
Mariner-1_Tbel (HSa) & 1,285 & $>30$ & $7.2 \pm 2(16)$ \\
TIGGER1 (Tbel) & 2,413 & $>80$ & $21.2 \pm 2(27)$ \\
TIGGER1 (EEu) & 2,410 & $>47$ & $28.0 \pm 3(25)$ \\
TIGGER1 (BT) & 2,408 & $>500$ & $17.3 \pm 2(102)$ \\
TIGGER1 (TTr) & 2,419 & $>580$ & $12.1 \pm 1(159)$ \\
Mariner-28_SIn (SIn) & 1,226 & Approximately 14 & $20.1 \pm 3(12)$ \\
Mariner1_BT (BT) & 1,277 & $>400$ & $14.7 \pm 2(95)$ \\
Mariner1_BT (TTr) & 1,285 & $>700$ & $7.6 \pm 1(101)$ \\
\hline
\end{tabular}

${ }^{a}$ The divergence represents average pairwise k-distance between individual copies and the consensus. Numbers of individual sequences used for the k-distance calculation are indicated in parentheses.

${ }^{\mathrm{b}}$ Mariner1_BT families from other Bos indicus, Bos grunniens, Bubalus bubalis are not shown. 


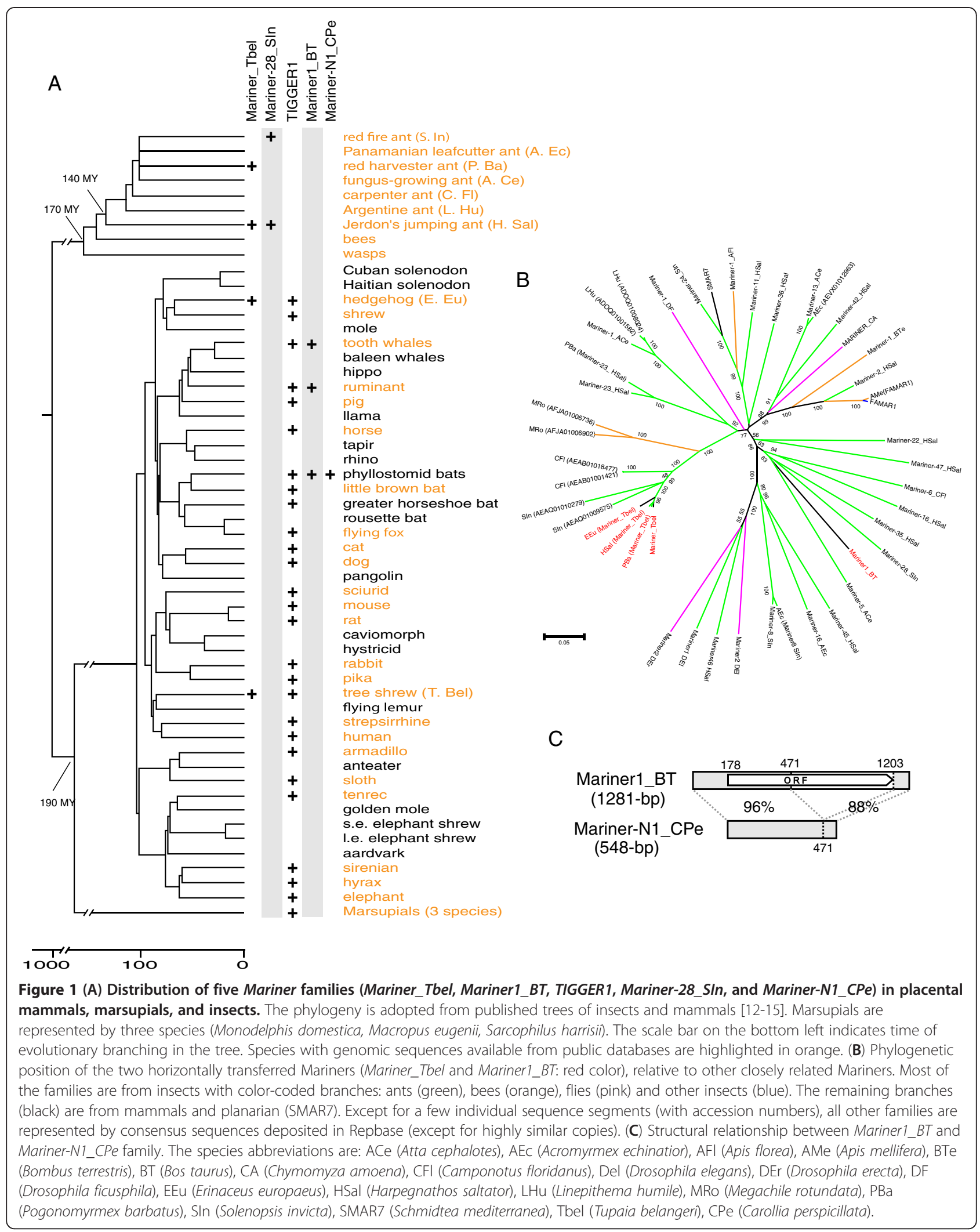


the ant and bee species (Figure 1A). Furthermore, Figure 1B indicates that the Mariner_Tbel family and many other similar Mariner families in ants and other insects shared a common ancestral sequence. These observations suggest the ancestor of ants Mariner_Tbel may have been present in some ant or other insect species very long time ago, probably as far back as the common ancestor of bees and ants (approximately 150 MYA) [14]. Thus, the mammalian Mariner_Tbel families probably originated from HTs from insects to mammals through some unknown vectors. Given that the two mammals belong to two distinct lineages, Mariner_Tbel in tree shrew and hedgehog may represent two independent HTs (Figure 1A). Notably, we cannot rule out the possibility that the Mariner_Tbel families in one of the two ant species, or both, also originated by HTs. This possibility is suggested by two facts: (a) the relatively young ages (at most approximately 43 to 50 MYA) of the two families, (b) the high identity (98.5\%) between the two family consensus sequences, even $H$. saltator and $P$. barbatus diverged from each other approximately 100 million years ago [13]. Among insect species, frequent HTs have been documented in flies [16]. Alternatively, Mariner_Tbel sequences could have survived for a very long time in either of the two ant genomes before the most recent family expansions.

In addition to mammalian Mariner_Tbel families, Mariner1_BT DNA families might also have originated by HT from insects (Figure 1A). We were able to obtain high quality consensus of Mariner1_BT from bovine species (Bos taurus, Bos indicus, Bos grunniens, Bubalus bubalis) and bottlenosed dolphin Tursiops truncatus (Table 2). All the derived consensus sequences show similar lengths (approximately 1,280 bp), and high pairwise identities throughout the entire length $(>98 \%)$. Blast screening against National Center for Biotechnology Information (NCBI) databases using Mariner1_BT consensus sequence as query also detected this family in several other mammalian species, including one bat species, Carollia perspicillata (Seba's short-tailed bat), additional ruminants, and whale (Table 3). These BlastN hits show similar score and query coverage ( $>80 \%$ identity to the consensus and $>90 \%$ coverage). In summary, Mariner1_BT type TEs were found only in three taxonomic groups to date: ruminants, tooth whales (Odontoceti), and New World leaf-nosed bats (Phyllostomidae) (Figure 1A). Notably, in C. perspicillata (short-tailed fruit bat) and Desmodus rotundus (vampire bat), we also detected a family of nonautonomous DNA transposon, called Mariner-N1_CPe, which was likely derived from the bat Mariner1_BT family (Figure 1C).

Remarkably, the other closest relatives of Mariner1_BT are all found in ant species: Mariner1_BT coclusters significantly (bootstrap $=83$ ) with three other ant Mariner families (Mariner-5_ACe, Mariner-28_SIn and Mariner35_HSal) (Figure 1B). Given the vast diversity of Mariners found in insects (Figure 1B), and the confined distribution of Mariner1_BT in mammals, we propose Mariner1_BT family could also originate from a horizontally transferred insect-like element. Using a similar method above, that is, based on the family divergence and mammalian phylogeny (Table 2 and Figure 1A), we estimated the ages of bovine Mariner1_BT to be 90 to 85 MYA, and 90 to 63 MYA for dophin T. truncatus Mariner1_BT family. The age of Mariner1_BT in bat could not be estimated due to insufficient data. We also could not determine if HT happened in mammals more than once, because the three taxonomic groups that include Mariner1_BT are relatively close.

In summary, this is the first report of two cases of horizontally transferred Mariner elements (Mariner_Tbel and Mariner1_BT) between insects and mammals. Previously, four families of DNA transposons from the $h A T$ superfamily were also found to be involved in multiple waves of HT between insects and other vertebrates including mammals [11]. This could partially be attributed to the fact that insects are the largest and the most diverse group of invertebrate animals on earth. While insects are the most likely source of the horizontally transferred transposons, the original source or possible intermediaries, such as parasitic insects [11] or viruses, remain unclear. This is complicated by the possibility that recurrent HTs of related Mariner elements are likely to take place between different insects [16]. The

Table 3 Mariner1_BT sequences detected in mammals

\begin{tabular}{llllllll}
\hline Groups & Species & Accession & Score & Query coverage & E value & Identity & Gaps \\
\hline Bat & Carollia perspicillata & AC152852.2 & 1,324 & $98 \%$ & 0 & $1,068 / 1,291(83 \%)$ & $87 / 1,291(7 \%)$ \\
Ruminants & Odocoileus hemionus & AY330343.1 & 1,278 & $98 \%$ & 0 & $1,061 / 1,294(82 \%)$ & $73 / 1,294(6 \%)$ \\
& Ovis aries & AC148039.3 & 1,274 & $99 \%$ & 0 & $1,078 / 1,309(82 \%)$ & $64 / 1,309(5 \%)$ \\
& Muntiacus reevesi & AC174385.3 & 1,265 & $99 \%$ & 0 & $1,074 / 1,312(82 \%)$ & $68 / 1,312(5 \%)$ \\
& Muntiacus muntjak vaginalis & AC152844.3 & 1,242 & $99 \%$ & 0 & $1,071 / 1,313(82 \%)$ & $71 / 1,313(5 \%)$ \\
& Capra hircus & EU870890.1 & 1,142 & $98 \%$ & 0 & $1,031 / 1,296(80 \%)$ & $109 / 1,296(8 \%)$ \\
\multirow{2}{*}{ Whale } & Pseudorca crassidens & AP011081.1 & 1,833 & $98 \%$ & 0 & $1,180 / 1,288(92 \%)$ & $22 / 1,288(2 \%)$ \\
\hline
\end{tabular}

The table shows only top scores using Mariner1_BT as BlastN query. 
role of viruses in HT proposed some time ago [17] still remains to be understood. As more genome sequence data become available, more mechanistic details on HT between mammals and insects are likely to emerge.

\section{Methods}

Mariner transposable elements from Repbase (http:// www.girinst.org/repbase/) were used as an initial query to screen Mariners in diverse genomes available at NCBI (National Center for Biotechnology Information: http:// www.ncbi.nlm.nih.gov/). Family consensus sequences were constructed whenever possible. The copy numbers in each family were determined by BLASTN using consensus sequences as queries. Sequence divergence within each family was assessed by the average pairwise kdistance (Kimura two-parameter model) between individual insertions and the corresponding consensus sequences. The k-distance was calculated using the software MEGA 4 [18]. For a given family, individual sequences used in k-distance calculation were randomly chosen from the family members; in most cases individual sequences matched $>70 \%$ of the consensus length.

We used Mariner_Tbel and Mariner1_BT as BLASTN queries against Repbase to select top scoring TE entries for phylogeny analysis. Individual sequences selected from GenBank were also used in the tree if Repbase consensus sequences were not available. The sequence alignments are shown in Additional file 1. The alignments were done using the online MAFFT server (http://mafft.cbrc.jp/alignment/software/). The phylogeny tree was inferred using MEGA 4 [18], using the neighbor joining (NJ) method and k-distances. Branch support was estimated using 1,000 bootstrap replicates.

\section{Additional file}

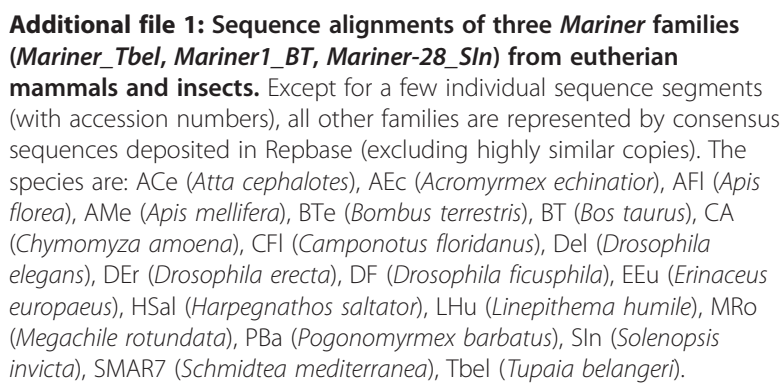

\section{Abbreviations}

ACe: Atta cephalotes; AEc: Acromyrmex echinatior; AFI: Apis florae; AMe: Apis mellifera; BT: Bos Taurus; BTe: Bombus terrestris; CA: Chymomyza amoena; CFI: Camponotus floridanus; Del: Drosophila elegans; DEr: Drosophila erecta; DF: Drosophila ficusphila; EEu: Erinaceus europaeus; HSal: Harpegnathos saltator; HT: Horizontal transfer; LHu: Linepithema humile; MRo: Megachile rotundata; PBa: Pogonomyrmex barbatus; SIn: Solenopsis invicta;

SMAR7: Schmidtea mediterranea; Tbel: Tupaia belangeri; Tr: Tursiops truncatus.

\section{Competing interests}

The authors declare that they have no competing interests.

\section{Authors' contributions}

SGO contributed to development of the hypothesis, collection, preparation, analysis and interpretation of data, wrote the first draft of the manuscript, and revised the text. WB contributed to the analysis and interpretation of data, writing and revising the manuscript. CM contributed to the discussion of data and revisions of the manuscript. J J contributed to development of the hypothesis, interpretation of data and final revisions. All the authors read and approved the final manuscript.

\section{Acknowledgements}

This work was supported by funds from the Sao Paulo Research Foundation (FAPESP), Sao Paulo State University (UNESP) and the National Institutes of Health grant 5 P41 LM006252. The content of this manuscript is solely the responsibility of the authors and does not necessarily represent the official views of the National Library of Medicine or the National Institutes of Health.

\section{Author details}

${ }^{1}$ Morphology Department, Bioscience Institute, UNESP - Sao Paulo State University, Botucatu, Sao Paulo 18618-970, Brazil. ${ }^{2}$ Genetic Information Research Institute, 1925 Landings Drive, Mountain View, CA 94043, USA.

Received: 11 May 2012 Accepted: 24 August 2012

Published: 26 September 2012

\section{References}

1. Syvanen M: Horizontal gene transfer: evidence and possible consequences. Annu Rev Genet 1994, 28:237-261.

2. Brown JR: Ancient horizontal gene transfer. Nat Rev Genet 2003, 4:121-132.

3. Hartl DL, Lohe AR, Lozovskaya ER: Modern thoughts on an ancyent marinere: function, evolution, regulation. Annu Rev Genet 1997, 31:337-358

4. Sanchez-Gracia A, Maside X, Charlesworth B: High rate of horizontal transfer of transposable elements in Drosophila. Trends Genet 2005, 21:200-203.

5. Schaack S, Gilbert C, Feschotte C: Promiscuous DNA: horizontal transfer of transposable elements and why it matters for eukaryotic evolution. Trends Ecol Evol 2010, 25:537-546.

6. Silva JC, Loreto EL, Clark JB: Factors that affect the horizontal transfer of transposable elements. Curr Issues Mol Biol 2004, 6:57-71.

7. Daniels SB, Peterson KR, Strausbaugh LD, Kidwell MG, Chovnick A: Evidence for horizontal transmission of the $\mathrm{P}$ transposable element between Drosophila species. Genetics 1990, 124:339-355.

8. Loreto EL, Valente VL, Zaha A, Silva JC, Kidwell MG: Drosophila mediopunctata P elements: a new example of horizontal transfer. J Hered 2001, 92:375-381.

9. Maruyama K, Hartl DL: Evidence for interspecific transfer of the transposable element mariner between Drosophila and Zaprionus. J Mol Evol 1991, 33:514-524.

10. Robertson HM, Lampe DJ: Recent horizontal transfer of a mariner transposable element among and between Diptera and Neuroptera. Mol Biol Evol 1995, 12:850-862.

11. Gilbert C, Schaack S, Pace JK 2nd, Brindley PJ, Feschotte C: A role for host-parasite interactions in the horizontal transfer of transposons across phyla. Nature 2010, 464:1347-1350.

12. Hedges SB, Blair JE, Venturi ML, Shoe JL: A molecular timescale of eukaryote evolution and the rise of complex multicellular life. BMC Evol Biol 2004, 4:2

13. Moreau CS, Bell CD, Vila R, Archibald SB, Pierce NE: Phylogeny of the ants: diversification in the age of angiosperms. Science 2006, 312:101-104

14. Brady SG, Larkin L, Danforth BN: Bees, ants, and stinging wasps (Aculeata). In In The Timetree of Life. Edited by Hedges SB, Kumar S. Oxford, UK: Oxford University Press; 2009:264-269.

15. Meredith RW, Janečka JE, Gatesy J, Ryder OA, Fisher CA, Teeling EC, Goodbla A, Eizirik E, Simão TL, Stadler T, Rabosky DL, Honeycutt RL, Flynn JJ, Ingram CM, Steiner C, Williams TL, Robinson TJ, Burk-Herrick A, Westerman M, Ayoub NA, Springer MS, Murphy WJ: Impacts of the cretaceous terrestrial revolution and $\mathrm{KPg}$ extinction on mammal diversification. Science 2011, 334:521-524. 
16. Bartolome C, Bello X, Maside X: Widespread evidence for horizontal transfer of transposable elements across Drosophila genomes. Genome Biol 2009, 10:R22.

17. Kidwell MG: Lateral transfer in natural populations of eukaryotes. Annu Rev Genet 1993, 27:235-256.

18. Tamura K, Dudley J, Nei M, Kumar S: MEGA4: Molecular Evolutionary Genetics Analysis (MEGA) software version 4.0. Mol Biol Evol 2007, 24:1596-1599

doi:10.1186/1759-8753-3-14

Cite this article as: Oliveira et al:: Horizontal transfers of Mariner transposons between mammals and insects. Mobile DNA 2012 3:14

\section{Submit your next manuscript to BioMed Central} and take full advantage of:

- Convenient online submission

- Thorough peer review

- No space constraints or color figure charges

- Immediate publication on acceptance

- Inclusion in PubMed, CAS, Scopus and Google Scholar

- Research which is freely available for redistribution 